\title{
Fosfomycin in the treatment of extended spectrum beta-lactamase- producing Enterobacteriaceae- related urinary tract infections
}

\author{
Ayhanım Tumturk ${ }^{1}$, Senol Tonyali ${ }^{2}$, Ayse Yasemin Tezer Tekce ${ }^{1}$, Levent Isikay ${ }^{2}$, Hakan Cime $^{3}$ \\ 1 University of Health Sciences, Clinic of Infectious Diseases, Turkiye Yuksek Ihtisas Training and Research \\ Hospital, Ankara, Turkey \\ 2 University of Health Sciences, Clinic of Urology, Turkiye Yuksek Ihtisas Training and Research Hospital, Ankara, \\ Turkey \\ ${ }^{3}$ University of Health Sciences, Turkiye Yuksek Ihtisas Training and Research Hospital, Ankara, Turkey
}

\begin{abstract}
Introduction: We aimed to demonstrate if fosfomycin tromethamine (FT) treatment could be the treatment of choice in ESBL-producing Enterobacteriaceae strains as an alternative to carbapenem particularly in patients who we would like to treat on an outpatient basis.

Methodology; We retrospectively analyzed the medical records of all patients who admitted to infectious disease outpatient clinic with complaints of dysuria and frequency and received FT for lower UTI between May 2016 and May 2017.

Results: A total of 48 patients, 19 females (39.6\%) and 29 males (60.4\%), with a mean age of 62.5 (ranging from 27 to 85 ) years were included the study. $26(76.4 \%)$ of patients with a history of urinary operation or intervention had also a history of antibiotic use within the past 3 months. The isolated pathogens included Escherichia Coli $(\mathrm{n}=32)$, Klebsiella spp. $(\mathrm{n}=12)$, Enterobacter spp. $(\mathrm{n}=4)$. The overall microbiological response after treatment was $70.8 \%(34 / 48)$ and the clinical response was $75 \%(36 / 48)$. Clinical and microbiological response rates of patients with and without urinary operation/intervention, diabetes mellitus, history of antibiotic use and malignancy were found similar ( $\mathrm{p}>0.05$ ). However, patients with a urinary stone disease history had significantly higher response rates than those without a urinary stone disease history $(\mathrm{P}=0.042)$.

Conclusion: Oral fosfomycin tromethamine might be the treatment of choice in ESBL-producing enterobactericea related UTIs especially caused by Escherichia Coli.
\end{abstract}

Key words: urinary tract infection; fosfomycin; enterobacteriaceae; Escherichia coli; Klebsiella.

J Infect Dev Ctries 2019; 13(1):73-76. doi:10.3855/jidc. 10658

(Received 04 July 2018 - Accepted 28 November 2018)

Copyright (C) 2019 Tumturk et al. This is an open-access article distributed under the Creative Commons Attribution License, which permits unrestricted use, distribution, and reproduction in any medium, provided the original work is properly cited.

\section{Introduction}

Urinary tract infections (UTIs) are one of the most common infections both in inpatient and outpatient populations. The majority of these infections are uncomplicated lower UTIs. UTIs caused by Enterobacteriaceae, which produces extended spectrum beta-lactamase (ESBL), are seen all over the world as well as increasing frequency in our country [1,2]. For community-associated ESBL infection; previous antibiotic use, recurrent UTIs, diabetes mellitus, urinary instrumentation, female sex and being over 65 years old are considered as risk factors [3]. However, it is reported that in recent years there has been an increase in the frequency of community-acquired infections due to ESBL-producing Enterobacteriaceae isolates in which no risk factor could be detected [4]. Carbapenems are the most reliable antibiotics for infections caused by ESBL producing
Enterobacteriaceae. However, overuse of these drugs causes the problem of increased drug resistance [5]. Treatment with these antibiotics requires patients to be hospitalized and also increases the cost of treatment [1]. There are a small number of oral antibiotics available in the empirical treatment of UTIs caused by ESBLproducing pathogens. Large-scale isolate investigations have shown that many isolates of Escherichia coli responsible for UTIs are resistant to most oral antibiotics, including fluoroquinolones, trimethoprimsulfamethoxazole (TMP-SMX) and $\beta$-lactam agents [6]. Fosfomycin tromethamine (FT), a naturally occurring antimicrobial agent, exhibits broad-spectrum antibacterial activity by suppressing the peptidoglycan, an important component of the bacterial cell wall, synthesis pathway of Gram-negative pathogens [7]. FT has good antimicrobial activity against clinical isolates of ESBL producing Enterobacteriaceae [8]. 
FT is an effective, inexpensive and easily applicable therapy that can be used in the treatment of uncomplicated lower UTIs infections caused by ESBLproducing Enterobacteriaceae strains.

Although there are many publications about the in vitro activity of the FT on Enterobacteriaceae group, there is a scarce of data showing in vivo efficacy especially in patients with underlying risk factors. The aim of this study is to demonstrate if FT treatment could be the treatment of choice in ESBL-producing Enterobacteriaceae strains as an alternative to carbapenem particularly in patients who we would like to treat on an outpatient basis.

\section{Methodology}

After the approval of institutional review board, we retrospectively analyzed the medical records of all patients who admitted to infectious disease outpatient clinic with complaints of dysuria and frequency and received Fosfomycin tromethamine for lower UTI between May 2016 and May 2017. A total of 48 patients with $>20$ leukocytes $/ \mathrm{mm}^{3}$ in urine analysis, documented ESBL-producing Enterobactericea $\left(>10^{5}\right.$ $\mathrm{CFU} / \mathrm{mm}^{3}$ ) in urine culture and no fever or leukocytosis were included the study. Patients with a history of allergy to Fosfomycin tromethamine, younger than 18 years of old or who used concurrent antibiotics for the isolated organism were excluded from the study.

Clean-catch urine samples obtained from patients were inoculated on 5\% sheep blood agar and eosinmethylene blue agar by means of a $0.01 \mathrm{~mL}$ calibrated loop. Isolated strains were identified by the BD Phoenix automated system and sensitivity tests were performed. Antibiotic susceptibilities of isolates were determined according to CLSI (Clinical and Laboratory Standards Institute) recommendations [9]. ESBL productions were investigated by double disk synergy method; the minimum inhibitory concentration (MIC) values of Fosfomycin tromethamine were determined by E-test. According to CLSI criteria, those with MIC values $\geq$ $256 \mu \mathrm{g} / \mathrm{mL}$ were considered as resistant and those with $\leq 64 \mu \mathrm{g} / \mathrm{mL}$ were considered as sensitive.

Examined parameters included patients' age, sex, operation history, presence of underlying disease, history of antibiotic use in the last 3 months and clinical findings. A total of 2 doses of Fosfomycin tromethamine sachet (Monurol, Zambon S.p.A-Bilim İlaç or Uromisin, Koçak Farma İlaç) $(3 g \times 1)$ every other night were given to all patients. Clinical success was defined as symptom relief at the control visit and microbiological success was defined as a sterile control urine culture taken 7 to 9 days after the last dose of the
Table 1. Risk factors for urinary tract infection in the patient cohort.

\begin{tabular}{lcc}
\hline Risk factor & n & $\mathbf{\%}$ \\
\hline Urinary intervention/catheterization & 34 & 70.8 \\
Antibiotic usage in the past 3 months & 34 & 70.8 \\
Diabetes mellitus & 13 & 27.0 \\
Nephrolithiasis & 10 & 20.8 \\
Malignancy involving the urinary tract & 7 & 14.6 \\
Other malignancies & 2 & 4.1 \\
\hline
\end{tabular}

drug according to the guidelines of the American Society of Infectious Diseases (ASID) guidelines [10].

Patients' urine cultures performed at first week and one month after treatment was also examined. In addition, the urine culture results of the patients who had readmitted to the hospital were evaluated.

Isolation of ESBL-producing Enterobacteriaceae in the control urine culture within 28-30 days posttreatment was defined as relapse. Reinfection was defined as isolation of any pathogens in the urine culture within 28-30 days post-treatment.

\section{Results}

A total of 48 patients, 19 females (39.6\%) and 29 males $(60.4 \%$ ), with a mean age of 62.5 (ranging from 27 to 85 ) years were included the study. Among 48 patients, 37 patients were older than 50 years old. 34 patients $(70.8 \%)$ had a history of either urinary operation or intervention, of which 31 (91.1\%) were older than 50 years. $26(76.4 \%)$ of patients with a history of urinary operation or intervention had also a history of antibiotic use within the past 3 months (Table 1). Previous urinary operation/catheterization and antibiotic use within the past 3 months were the most common predisposing factors for ESBL-producing Enterobactericae related lower UTIs.

The isolated pathogens included E. coli $(\mathrm{n}=32)$, Klebsiella spp. $(\mathrm{n}=12)$, Enterobacter spp. $(\mathrm{n}=4)$. All isolates were resistant to ciprofloxacin and TMP/SMX while were sensitive to FT, imipenem / cilastatin, meropenem and ertapenem.

The overall microbiological response after treatment was $70.8 \%(34 / 48)$ and the clinical response was $75 \%(36 / 48)$. When the subgroup analysis of this result was performed, the microbiological response rate in patients with Klebsiella spp-related infection was $50 \%(6 / 12)$, whereas $79 \%(25 / 32)$ in patients with E. Coli-related infection. Control urine culture performed 28 days after the antibiotherapy was available in 32 of 48 patients $(66.6 \%)$. Relapse and reinfection rates were $3.1 \%(2 / 32)$ and $9.3 \%(3 / 32)$, respectively. $85.7 \%$ 
$(12 / 14)$ of the patients with either relapse or reinfection had history of previous operation and antibiotic use.

Clinical and microbiological response rates of patients with and without urinary operation/intervention, diabetes mellitus, history of antibiotic use and malignancy were found similar $(\mathrm{p}>$ $0.05)$. However, patients with a urinary stone disease history had significantly higher response rates than those without a urinary stone disease history $(\mathrm{P}=$ $0.042)$.

\section{Discussion}

Urinary tract infections (UTIs) are commonly seen and constitute a significant burden of hospitalizations and health-care cost. Increased antimicrobial resistance and limited treatment options in Gram-negative bacteria have increased the interest in 'old' antibiotic FT [11]. FT is a phosphonic acid derivative. It has a broad-spectrum antibacterial activity, including Gram-positive and ESBL-producing gram-negative strains. FT has good in vitro activity against many multidrug resistant uropathogen species [12]. FT is an attractive drug in the treatment of UTI by means of certain properties including rapid absorption after oral administration and achieving high urine concentration, biofilm activity and efficacy against various multidrug-resistant organism such as extended spectrum beta-lactamase- (ESBL) and AmpC beta-lactamase- (AmpC) producing Enterobacteriaceae. Oral FT is well tolerated and has no serious side effects. Only $5 \%$ of patients are reported to have side effects, most commonly diarrhea [11-14].

The most reliable treatment option for ESBLproducing Enterobactericae is carbapenem. But the widespread use of carbapenems brings with the problem of increased resistance [5]. None of the patients included in the present study were sensitive to ciprofloxacin and TMP/SMX, but were sensitive to FT and carbapenems. Oral FT treatment were given to outpatient clinic patients without fever and leukocytosis because carbapenem therapy needed patient hospitalization and was not cost-effective.

In the present study, oral FT treatment was more effective in ESBL-producing E. coli compared to Klebsiella spp., 79\% versus 50\%. In a study by Fedrigo et al, FT was reported to represent high in vitro activity against E. coli, as well as higher MIC distribution against Klebsiella spp. They suggested that this finding was in concordance with previous studies reporting standard single dose FT treatment inadequate for urinary tract infections caused by Klebsiella [14]. In a review by Flagas et al., among ESBL-producing Enterobactericea especially $E$. coli was found to be most sensitive to FT and it was emphasized that there is pre-clinical evidence showing that phosphomycin is a valuble treatment option in lower urinary tract infections caused by ESBL-producing E. coli [8]. In the present study, although oral FT treatment was initiated according to the in vitro susceptibility tests, half of the 12 patients who had ESBL-producing Klebsiella spp. continued to have ESBL-producing Klebsiella spp. in their urine cultures after FT treatment. This might suggest that invitro and invivo efficacy of FT might not be the same in ESBL-producing Klebsiella spp.-related UTIs.

Comorbidities and underlying diseases might also have an impact on treatment outcomes. In a retrospective cohort study by Matthews et al., no association was found between co-morbidities and FT treatment failure [13]. Our hospital is a tertiary referral center and it was difficult to treat most of the patients because of the underlying predisposing factors. However, no statistically significant association was found between the presence or absence of risk factors and treatment failure in accordance with the aforementioned study. This might be indicative of FT may also be effective in the treatment of such difficult patients.

In a retrospective cohort study by Veve et al. [15] comparing oral FT with ertapenem in ESBL-producing bacteria-related urinary tract infections, responses to oral FT treatment were not lower than intravenous ertapenem treatment. Similarly in another study Şenol et al. found that the clinical and microbiologic response rates were comparable in oral FT and i.v. carbapenem treatments [16].

In the present study, oral Fosfomycin tromethamine treatment represents high clinical and microbiological success of treatment in UTIs caused by ESBLproducing enterobactericea, especially E. coli, including patients with underlying predisposing factor without fever and leucocytosis. However, despite the in-vitro efficacy treatment success remains at $50 \%$ in infections caused by Klebsiella spp. Also none of the patients had an oral treatment alternative. Thus, FT seems to be a good treatment alternative as being costeffective and having low side effects and also it is without disadvantages such as hospitalization, time loss, etc. From a patient's point of view, oral outpatient antibiotherapy might be superior in terms of quality of life and might avoid the risk of infection or thrombosis due to catheterization [15]. 


\section{Conclusion}

As a result, oral Fosfomycin tromethamine might be the treatment of choice in ESBL-producing enterobactericea related UTIs especially caused by E. Coli. In addition, the presence of underlying predisposing factors is not a confounding factor for the use of oral FT.

\section{Author contributions}

A.T., L.I., S.T., Y.T.T., H.C.: Protocol development, A.T., L.I.,Y.T.T.: Data collection and experiments, A.T., S.T.,H.C.: Data analysis and interpretation, A.T., L.I., S.T., Y.T.T.: Study supervision, A.T., L.I., S.T., Y.T.T., H.C.: Manuscript writing/editing

\section{References}

1. Tasbakan MI, Pullukcu H, Sipahi OR, Yamazhan T, Ulusoy S (2012) Nitrofurantoin in the treatment of extended-spectrum beta-lactamase-producing Escherichia coli-related lower urinary tract infection. Int J Antimicrob Agents 40: 554-556.

2. Pullukcu H, Tasbakan M, Sipahi OR, Yamazhan T, Aydemir S, Ulusoy S (2007) Fosfomycin in the treatment of extended spectrum beta-lactamase-producing Escherichia coli-related lower urinary tract infections. Int J Antimicrob Agents 29: 6265.

3. Dhillon RH, Clark J (2012) ESBLs: A clear and present danger? Crit Care Res Pract 2012: 625170.

4. Doi Y, Adams J, O'Keefe A, Quereshi Z, Ewan L, Paterson DL (2007) Community-acquired extended-spectrum betalactamase producers, United States. Emerg Infect Dis 13: 1121-1123.

5. Sheu CC, Lin SY, Chang YT, Lee CY, Chen YH, Hsueh PR (2018) Management of infections caused by extendedspectrum beta-lactamase-producing Enterobacteriaceae: current evidence and future prospects. Expert Rev Anti Infect Ther 16: 205-218.

6. Linsenmeyer K, Strymish J, Weir S, Berg G, Brecher S, Gupta K (2016) Activity of fosfomycin against extended-spectrumbeta-lactamase-producing uropathogens in patients in the community and hospitalized patients. Antimicrob Agents Chemother 60: 1134-1136.

7. Bi W, Li B, Song J, Hong Y, Zhang X, Liu H, Lu H, Zhou T, Cao J (2017) Antimicrobial susceptibility and mechanisms of fosfomycin resistance in extended-spectrum beta-lactamaseproducing Escherichia coli strains from urinary tract infections in Wenzhou, China. Int J Antimicrob Agents 50: 29-34.

8. Falagas ME, Kastoris AC, Kapaskelis AM, Karageorgopoulos DE (2010) Fosfomycin for the treatment of multidrug-resistant, including extended-spectrum beta-lactamase producing,
Enterobacteriaceae infections: a systematic review. Lancet Infect Dis 10: 43-50.

9. Clinical and Laboratory standard institute (CLSI) (2007) Performance standards for antimicrobial susceptibility testing, $23^{\text {rd }}$ informational supplement. CLSI document M100-S23 (ISBN 1-56238-866-5).

10. Gupta K, Hooton TM, Naber KG, Wullt B, Colgan R, Miller LG, Moran GJ, Nicolle LE, Raz R, Schaeffer AJ, Soper DE, Infectious Diseases Society of A, European Society for M, Infectious D (2011) International clinical practice guidelines for the treatment of acute uncomplicated cystitis and pyelonephritis in women: A 2010 update by the Infectious Diseases Society of America and the European Society for Microbiology and Infectious Diseases. Clin Infect Dis 52: e103-120.

11. Fransen F, Hermans $\mathrm{K}$, Melchers MJB, Lagarde CCM, Meletiadis J, Mouton JW (2017) Pharmacodynamics of fosfomycin against ESBL- and/or carbapenemase-producing Enterobacteriaceae. J Antimicrob Chemother 72: 3374-3381.

12. Novelli A, Rosi E (2017) Pharmacological properties of oral antibiotics for the treatment of uncomplicated urinary tract infections. J Chemother 29: 10-18.

13. Matthews PC, Barrett LK, Warren S, Stoesser N, Snelling M, Scarborough M, Jones N (2016) Oral fosfomycin for treatment of urinary tract infection: a retrospective cohort study. BMC Infect Dis 16: 556.

14. Fedrigo NH, Mazucheli J, Albiero J, Shinohara DR, Lodi FG, Machado A, Sy SKB, Tognim MCB (2017) Pharmacodynamic evaluation of fosfomycin against Escherichia coli and Klebsiella spp. from urinary tract infections and the influence of $\mathrm{pH}$ on fosfomycin activities. Antimicrob Agents Chemother 61: e 02498-16.

15. Veve MP, Wagner JL, Kenney RM, Grunwald JL, Davis SL (2016) Comparison of fosfomycin to ertapenem for outpatient or step-down therapy of extended-spectrum beta-lactamase urinary tract infections. Int J Antimicrob Agents 48: 56-60.

16. Senol S, Tasbakan M, Pullukcu H, Sipahi OR, Sipahi H, Yamazhan T, Arda B, Ulusoy S (2010) Carbapenem versus fosfomycin tromethanol in the treatment of extended-spectrum beta-lactamase-producing Escherichia coli-related complicated lower urinary tract infection. J Chemother 22: 355-357.

\section{Corresponding author}

Senol Tonyali, MD, FEBU

Türkiye Yüksek Ihtisas Egitim ve Arastırma Hastanesi Uroloji

Klinigi 06230 Altındag Ankara Turkiye

Tel: +90 3123061829

Fax: +90 3123124120

E-mail: dr.senoltonyali@gmail.com

Conflict of interests: No conflict of interests is declared. 